\title{
Preventing Deterioration of Active Vibration Control Effect Due to Aging Deterioration and Damage based on Deep Learning
}

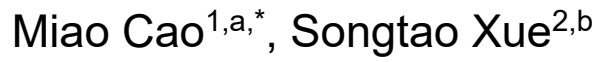 \\ ${ }^{1}$ Lect., Department of Architecture, Tohoku Institute of Technology, Japan \\ 2 Prof., Department of Architecture, Tohoku Institute of Technology, Japan \\ Prof., Department of Disaster Mitigation for Structures, Tongji University, China \\ acaomiao@tohtech.ac.jp, bxuest@tohtech.ac.jp
}

Keywords: Preventing Deterioration, Active Vibration Control, Deep Learning, Convolutional Neural Network, $\mathrm{H} \infty$ Control

\begin{abstract}
When designing a building's active vibration control, it is necessary to properly evaluate the effect on the control effect caused by changes in vibration characteristics due to aging deterioration and damage. However, although the previous designs have certain robustness against changes in vibration characteristics, they have insufficient on these control effect. In this paper, we establish a method based on deep learning to identify changes in vibration characteristics. Using this method, we can achieve to prevent reduction of the active control effect.
\end{abstract}

\section{Introduction}

In Japan, many buildings built after the period of high economic growth have already been shaken by numerous earthquakes, have become damaged and deteriorated, and are now under pressure to be repaired or renewed. Changes in vibration characteristics due to aging deterioration and damage have adverse effects on seismic-resistant buildings and seismic controlled buildings.

In particular, in the case of active control, control is tuned based on the state equation, which includes the vibration characteristics of the structure. Therefore, when aged deterioration or damage occurs, the state equation and the actual situation are inconsistent, which leads to a reduction in control effect.

To prevent such a reduction in control effect, we must identify the vibration

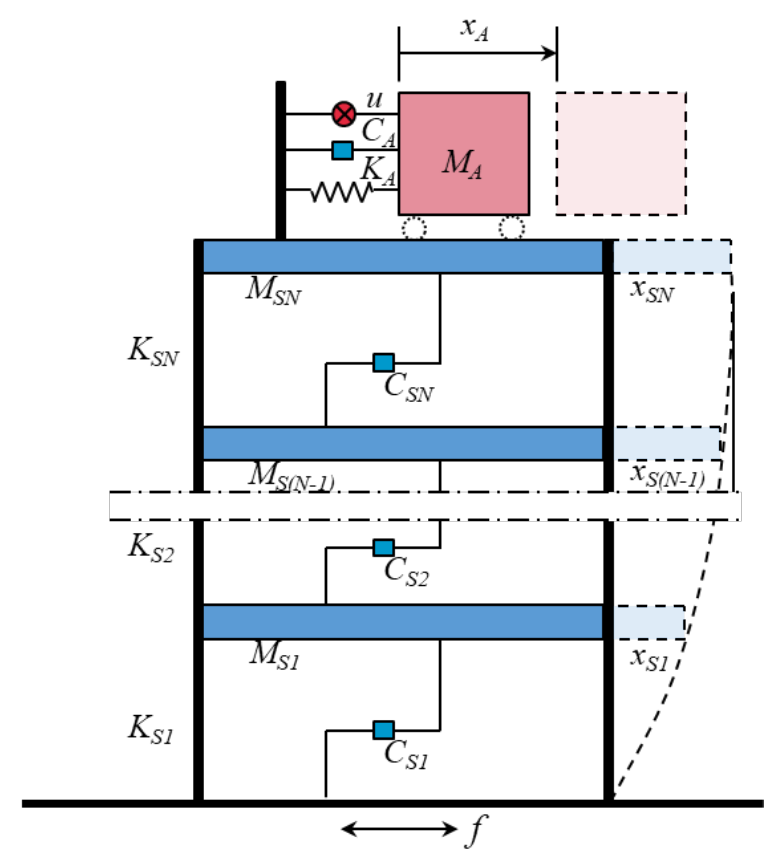

Figure 1 Analytical model of a MDF structure characteristics of the structure and reflect them in the equation of state. However, some reports show that the mainstream identification methods have the following problems: The identification accuracy of damping, which has a significant effect on active control, is unstable and varies 
widely [1], [2]. At present, although we can use a passive control device such as an oil damper jointly ${ }^{[3]}$ to prevent the reduction of the control effect due to such unexpected changes in vibration characteristics, a fundamental solution has not been achieved.

In this paper, we establish a method based on deep learning to identify changes in vibration characteristics due to aging deterioration and damage. Using this method, we can achieve to prevent reduction of the active control effect.

\section{Mathematical Model}

Figure 1 shows an analytical model of a multi-degree-of-freedom structure with active vibration control devices. The equation of motion corresponding to Fig. 1 is expressed by Eq. 1 .

$$
\left[\begin{array}{cc}
M_{A} & 0 \\
0 & M_{S}
\end{array}\right]\left\{\begin{array}{l}
\ddot{x}_{A} \\
\ddot{x}_{S}
\end{array}\right\}+\left[\begin{array}{cc}
C_{A} & -C_{A} \\
-C_{A} & C_{A}+C_{S}
\end{array}\right]\left\{\begin{array}{l}
\dot{x}_{A} \\
\dot{x}_{S}
\end{array}\right\}+\left[\begin{array}{cc}
K_{A} & -K_{A} \\
-K_{A} & K_{A}+C_{S}
\end{array}\right]\left\{\begin{array}{l}
x_{A} \\
x_{S}
\end{array}\right\}=\left\{\begin{array}{l}
f_{A} \\
f_{S}
\end{array}\right\}+\left\{\begin{array}{c}
-u \\
u
\end{array}\right\}
$$

$M_{S}, C_{S}$, and $K_{S}$ are symmetric matrices of $N \times N$ used to represent mass, damping coefficient, and stiffness coefficient. We convert the equation of motion into a state-space equation as Eq. 2 . Simultaneously, considering the versatility, we choose an electromagnetic force linear actuator as the active vibration control device. Therefore, the control force $u$ of the device is expressed by Eq. 3, where $I$ is the control current, and $\gamma, \sigma$ are the actuator coefficient.

$$
\begin{aligned}
& M \ddot{x}+C \dot{x}+K x=F f+U u \\
& u=\frac{\sigma}{\gamma} I+\frac{1}{\gamma} \dot{I}
\end{aligned}
$$

The state-space representation of our system is in the following equation.

$$
\left.\begin{array}{l}
\dot{X}=A X+B_{1} f+B_{2} u \\
Y=C_{1} X+D_{12} u
\end{array}\right\}
$$

where

$$
\begin{aligned}
& X=\left\{x_{S 1}, \dot{x}_{S 2}^{\cdot}, \cdots, x_{S N}^{\cdot}, \dot{x_{A}}, x_{S 1}, x_{S 2}, \cdots, x_{S N}, x_{A}, I\right\}^{T} \\
& A=\left[\begin{array}{ccc}
-M^{-1} C & -M^{-1} K & 0_{N+1,1} \\
I_{N+1, N+1} & 0_{N+1, N+1} & 0_{N+1,1} \\
0_{1, N+1} & 0_{1, N+1} & -\sigma
\end{array}\right] \\
& B_{1}=\left[\begin{array}{c}
M^{-1} F \\
0_{N+2, N+1}
\end{array}\right] \\
& B_{2}=\left[\begin{array}{c}
M^{-1} U \\
0_{N+1,1} \\
\gamma
\end{array}\right]
\end{aligned}
$$

$C_{1}$ and $D_{12}$ are observation matrixes with $2 N+3$ rows and $n$ columns ( $n$ is the number of observations). We show the above relationship in the block diagram of state feedback control, as Fig. 2.

According to the $\mathrm{H} \infty$ control theory, we assume that the feedback control law conforms to the following equation ${ }^{[4]}$.

$$
u=-\Sigma^{-1} \Theta \mathrm{X}
$$




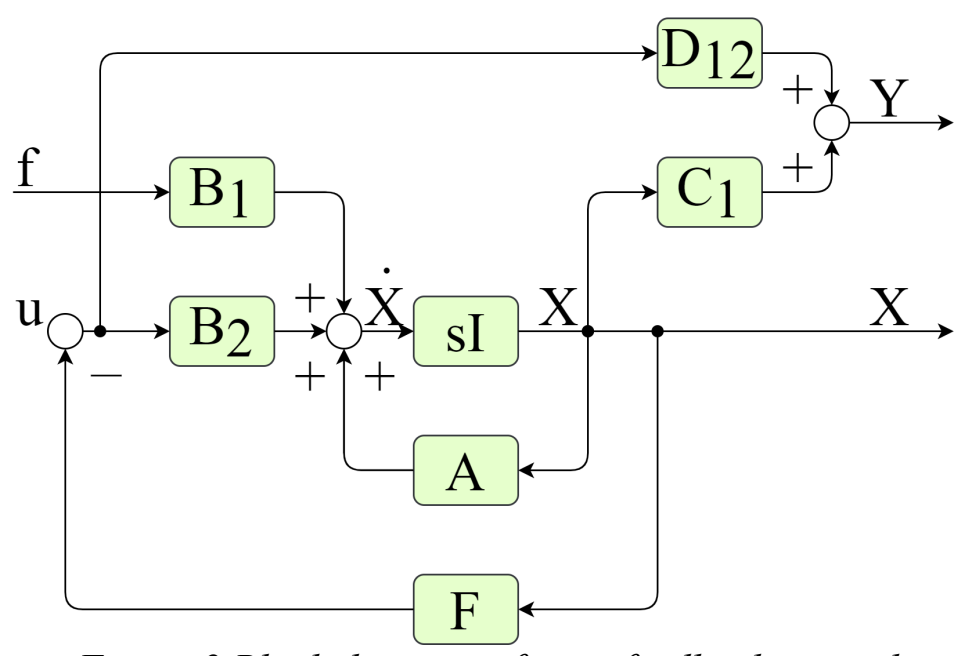

Figure 2 Block diagram of state feedback control

where

$$
\left.\begin{array}{l}
\Sigma=D_{12}^{T} D_{12} \\
\Sigma=D_{12}^{T} C_{1}+B_{2}^{T} P
\end{array}\right\}
$$

We can calculate $P$ by solving the following Riccati equation to obtain the optimal feedback control law.

$$
R^{T} P+P R-P S P+Q=0
$$

where

$$
\left.\begin{array}{l}
R=A-B_{2} \Sigma^{-1} D_{12}^{T} C_{1} \\
S=B_{1} B_{1}^{T}-B_{2} \Sigma^{-1} B_{2}^{T} \\
Q=C_{1}^{T} C_{1}-C_{1}^{T} D_{12} \Sigma^{-1} D_{12}^{T} C_{1}
\end{array}\right\}
$$

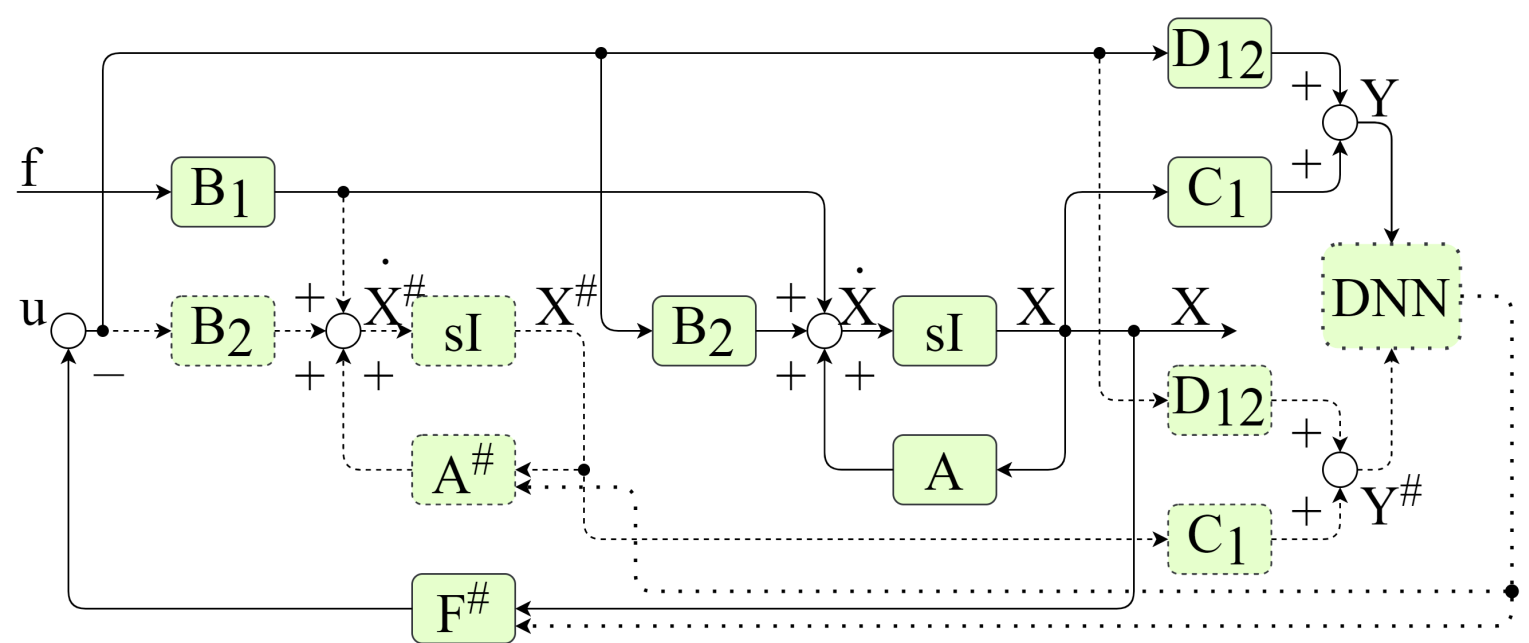

Figure 3 Block diagram of state feedback control based on deep learning when vibration characteristics changed 
This optimal feedback control law can play a significant role when the vibration characteristics of the structure we obtain are accurate. However, if the vibration characteristics change due to aging degradation or damage over time, the control status will be shown by the solid line in Fig. 3.

Here, the index \# in the symbol means the vibration characteristics before the change. In this status, we calculate the control force $u$ through an inaccurate feedback control law. Therefore, the control effect we get is not optimal, and may even have the opposite effect.

Although we can use some methods such as the Kalman filter, it requires the assumption that error information is known in advance, the accuracy of identification is not impeccable in this situation. Therefore, we try to use deep learning to identify accurate vibration characteristics. As shown in Figure 3, we compare the output before and after the change and input them into a convolutional neural network $(\mathrm{CNN})$ shown in Fig. 4.

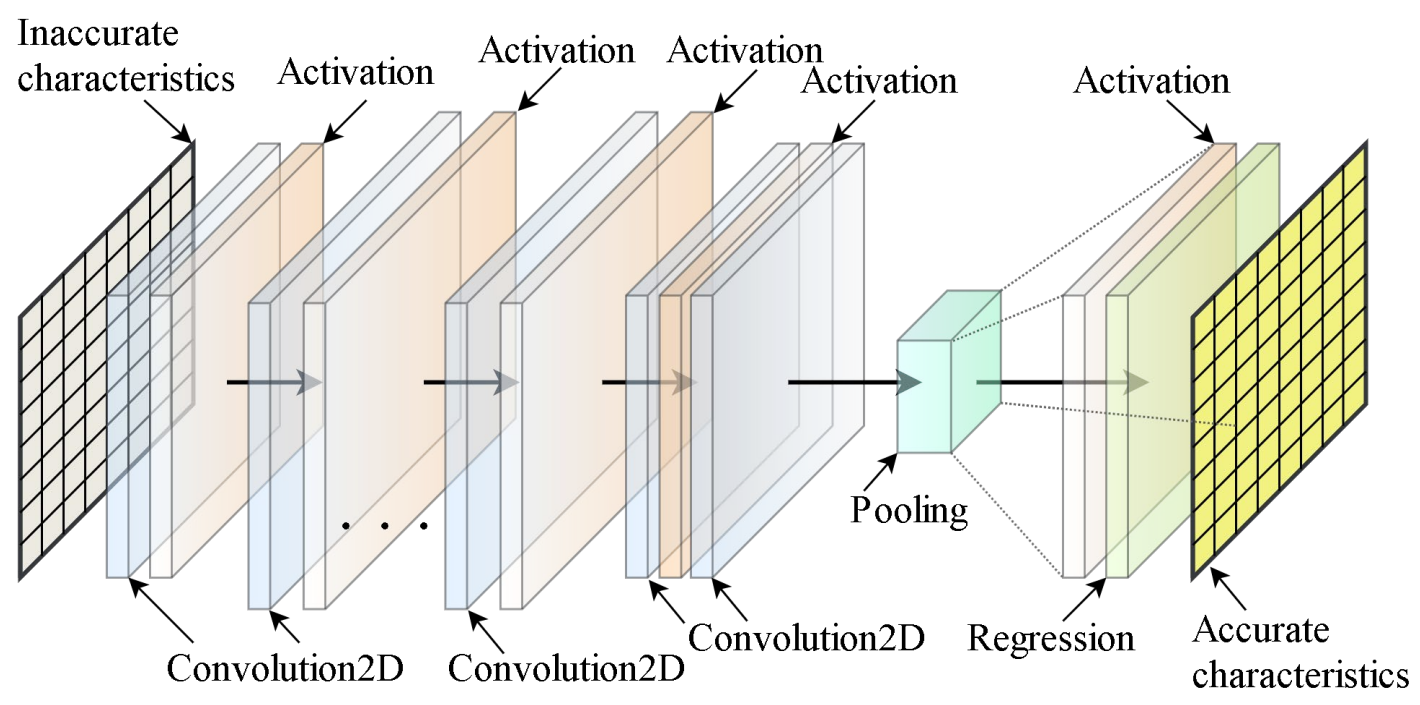

Figure 4 CNN architecture for identifying vibration characteristics

We use unsupervised learning ${ }^{[5]}$ to adjust the parameter in the neural network. The regression losses function is shown in Eq. 13.

$$
L(x)=\sqrt{\frac{1}{L} \sum_{i=1}^{L}\left\{Y[\varphi(x)]^{\#}-Y\right\}^{2}}
$$

where $L$ is the input ground motion duration and $\varphi$ is the output function of the CNN. We can also use Eq. 14 to calculate the half mean square error between the result of the CNN and the accurate characteristics to evaluate the learning effect after training.

$$
E(x)=\frac{1}{2} \sum \sum\{\varphi(x)-\Phi\}^{2}
$$

where $\Phi$ are the accurate value of vibration characteristics changed due to degradation and damage. 


\section{Numerical study}

We use a multi-degree-of-freedom structure simulation to confirm the effect of the proposed method. The structural characteristics of the model are shown in Table 1.

We use the Rayleigh damping shown in Eq. 15 as the damping coefficient of the structure. The damping coefficient of the AMD is set to the optimum damping, according to Eq 16.

\section{Table 1 Structural characteristics of model}

\begin{tabular}{|c|c|c|c|}
\hline Story & Mass[kg] & Stiffness $[\mathrm{N} / \mathrm{m}]$ & Damping \\
\hline 1 & 2000 & 250000 & \multirow{4}{*}{$\begin{array}{c}\text { Rayleigh Damping, } \\
{ }_{1} \mathrm{~h}=0.02, \\
{ }_{2} \mathrm{~h}=0.02\end{array}$} \\
\hline 2 & 2000 & 250000 & \\
\hline 3 & 2000 & 250000 & \\
\hline 4 & 2000 & 250000 & \\
\hline AMD & 320 & 62500 & Optimal tuning \\
\hline
\end{tabular}

$$
\left.\begin{array}{rl}
{[C]} & =a_{0}[M]+a_{1}[K] \\
a_{0} & =2{ }_{1} \omega \cdot{ }_{2} \omega\left({ }_{1} h_{2} \omega-{ }_{2} h{ }_{1} \omega\right) /\left({ }_{2} \omega^{2}-{ }_{1} \omega^{2}\right) \\
a_{1} & =2\left({ }_{2} h{ }_{2} \omega-{ }_{1} h{ }_{1} \omega\right) /\left({ }_{2} \omega^{2}-{ }_{1} \omega^{2}\right)
\end{array}\right\}
$$

where $\omega$ is the natural circular frequency of the structure.

$$
h_{\text {opt }}=\sqrt{\frac{3 \mu}{8(1+\mu)}}
$$

where $\mu$ is the mass ratio of the AMD and the structure.

We generate an artificial seismic wave by the non-stationary Kanai-Tajimi model ${ }^{[6]}$ shown in Eq. 17 as the input ground motion.

$$
G(\omega)=\frac{1+4 \eta^{2}\left(\frac{\omega}{\omega_{g}}\right)^{2}}{\left(1-\frac{\omega^{2}}{\omega_{g}^{2}}\right)^{2}+4 \eta^{2}\left(\frac{\omega}{\omega_{g}}\right)^{2}} G_{0}
$$

where $\omega_{p}$ is the unique peak of the spectrum, here is $0.5, \eta$ is the sharpness of the peak, and $G_{0}$ is the constant power spectral intensity of the excitation. Fig. 5 shows the Fourier spectrum and the acceleration record of the input ground motion.

The dynamic response of the $4^{\text {th }}$ story with and without the AMD is shown in Fig. 6. We have confirmed that the effect of the AMD is very significant from Fig. 6. Nevertheless, when the structural characteristics change, the AMD control effect will become shown in Fig. 7. According to the conclusions of references [7] and [8], we can know that the structure's stiffness reduction factor can reach 0.5 or less after a big earthquake. Therefore, we assume the stiffness reduction factor of the whole model to be 0.5. Comparing Fig. 6 and Fig. 7, we find that the dynamic response becomes large. That is because the structure's first natural frequency is closer to the input ground motion's dominant frequency. Although we expect the AMD to provide a vibration reduction effect, the AMD makes the structure's response larger. In other words, the AMD, which can reduce vibration, brings a harmful effect on the structure after vibration characteristics changed. Consequently, when aged deterioration and damage occurs, it leads to a reduction of the control effect. 

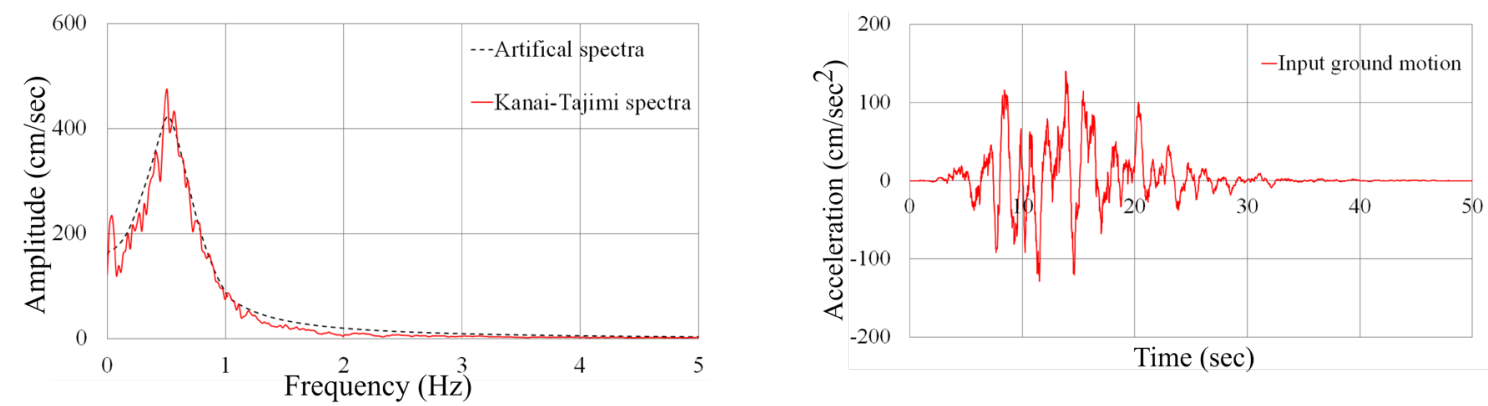

Figure 5 Input ground motion, (left) Fourier spectrum, (right) Acceleration records
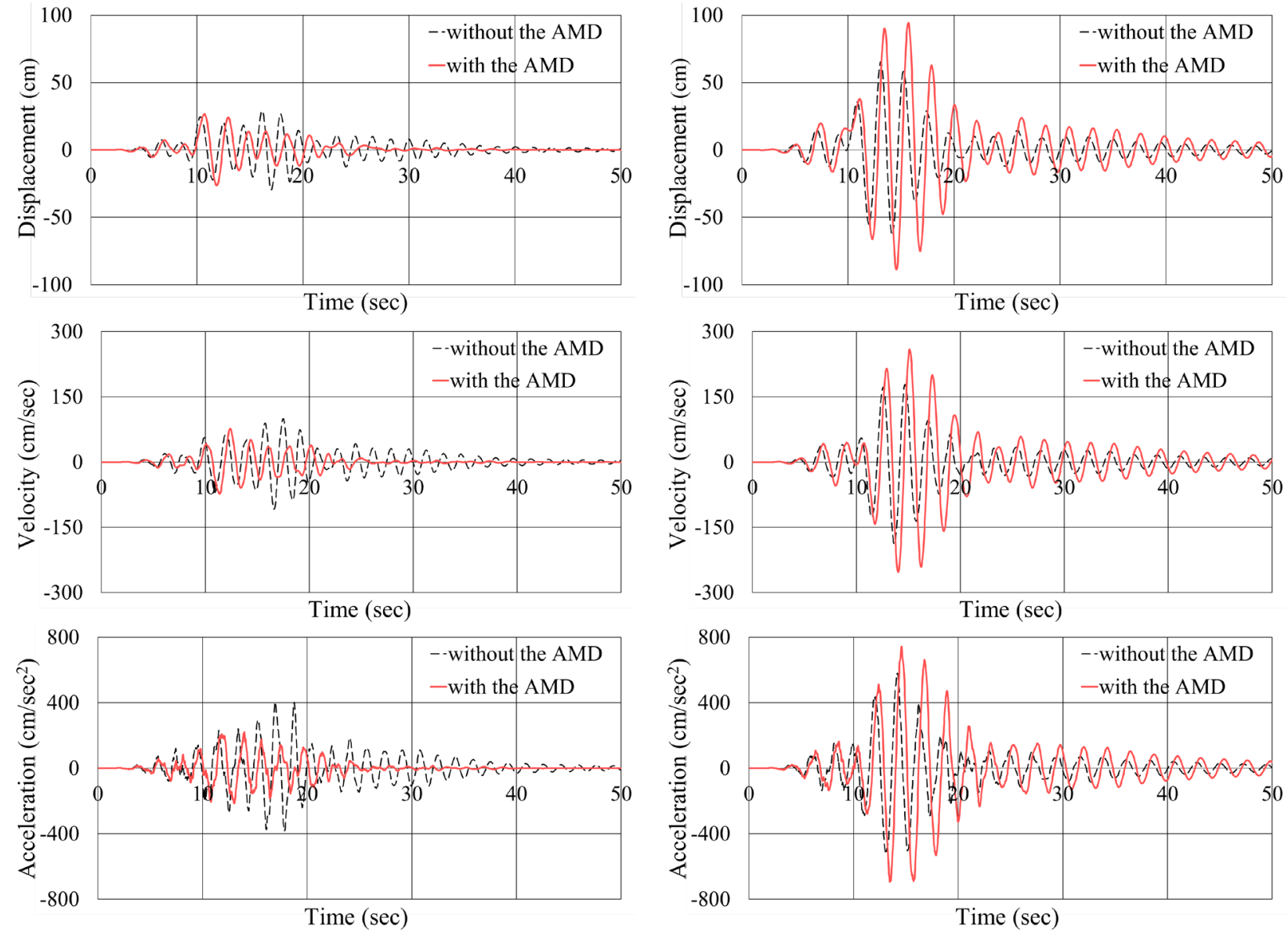

Figure 6 Dynamic response before vibration characteristics changed

Figure 7 Dynamic response after vibration characteristics changed

We create a convolutional neural network, whose architecture is shown in Table 2, and train it to estimate the accurate value of vibration characteristics. Fig. 8 shows the training regression loss of dynamic response and the heteroscedasticity-adjusted mean squared error(HMSE) of vibration characteristics. We stop the training after 2000 epochs because the results are excellent.

The dynamic response of the $4^{\text {th }}$ story with and without the AMD after training is shown in Fig. 9. Comparing with Fig. 7, the control effect of the AMD has increased significantly. Comparing the optimal results of the AMD when using reduced vibration characteristics as the original parameter, we find that the two results are precisely the same. It proves that we can maintain the optimal control effect by using our method even if the structure's vibration characteristics are changed. 
Table 2 Architecture of the CNN

\begin{tabular}{|c|c|c|c|c|}
\hline Layer & Type & Activations & Learnable Parameters & Total Learnable Parameters \\
\hline 1 & Input & $5 \times 1 \times 1$ & - & $\overline{0} 0$ \\
\hline 2 & Convolution & $5 \times 1 \times 16$ & $\begin{array}{ll}\text { Weights } & 3 \times 1 \times 1 \times 16 \\
\text { Bias } & 1 \times 1 \times 16\end{array}$ & 64 \\
\hline 3 & Tanh & $5 \times 1 \times 16$ & - & 0 \\
\hline 4 & Convolution & $5 \times 1 \times 32$ & $\begin{array}{l}\text { Weights } 3 \times 1 \times 16 \times 32 \\
\text { Bias } 1 \times 1 \times 32\end{array}$ & 1568 \\
\hline 5 & Tanh & $5 \times 1 \times 32$ & - & 0 \\
\hline 6 & Convolution & $5 \times 1 \times 64$ & $\begin{array}{l}\text { Weights } 3 \times 1 \times 32 \times 64 \\
\text { Bias } 1 \times 1 \times 64\end{array}$ & 6208 \\
\hline 7 & Tanh & $5 \times 1 \times 64$ & - & 0 \\
\hline 8 & Convolution & $5 \times 1 \times 32$ & $\begin{array}{l}\text { Weights } 3 \times 1 \times 64 \times 32 \\
\text { Bias } 1 \times 1 \times 32\end{array}$ & 6176 \\
\hline 9 & Tanh & $5 \times 1 \times 32$ & - & 0 \\
\hline 10 & Convolution & $5 \times 1 \times 16$ & $\begin{array}{ll}\text { Weights } & 3 \times 1 \times 32 \times 16 \\
\text { Bias } & 1 \times 1 \times 16\end{array}$ & 1552 \\
\hline 11 & Tanh & $5 \times 1 \times 16$ & - & 0 \\
\hline 12 & Convolution & $5 \times 1 \times 4$ & $\begin{array}{l}\text { Weights } 3 \times 1 \times 16 \times 4 \\
\text { Bias } 1 \times 1 \times 4 \\
\end{array}$ & 196 \\
\hline 13 & Tanh & $5 \times 1 \times 4$ & - & 0 \\
\hline 14 & Convolution & $5 \times 1 \times 1$ & $\begin{array}{l}\text { Weights } 3 \times 1 \times 4 \\
\text { Bias } 1 \times 1\end{array}$ & 13 \\
\hline 15 & Tanh & $5 \times 1 \times 1$ & - & 0 \\
\hline 16 & Regression & & - & 0 \\
\hline
\end{tabular}
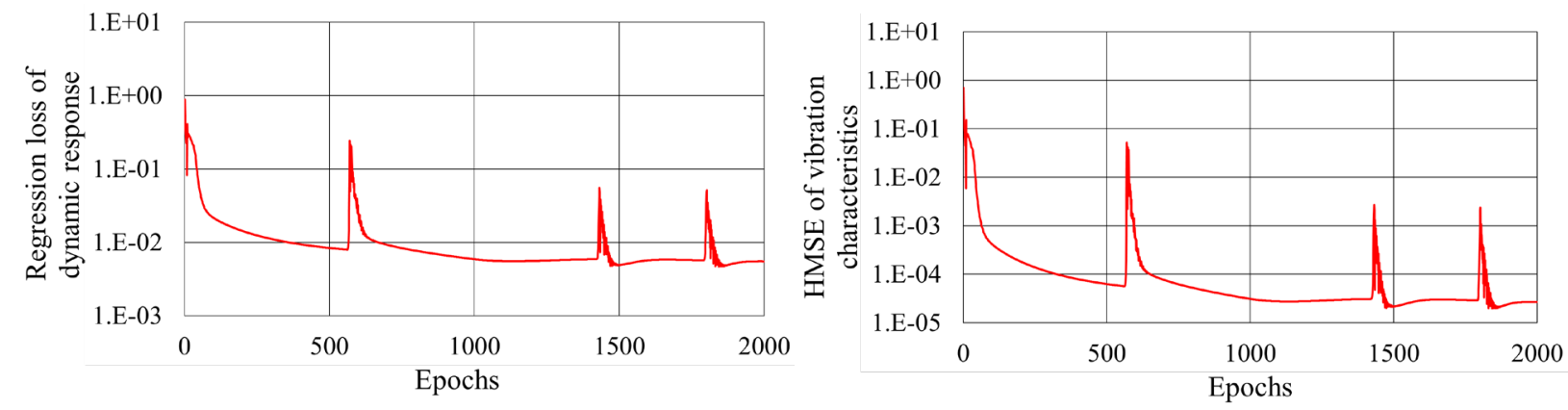

Figure 8 Training regression loss and the HMSE

\section{Summary}

We discussed the changes in the state-space representation of a structure-AMD system before and after the vibration characteristics change due to aging deterioration and damage. After the vibration characteristics change, the AMD, which was tuned to the optimal control by us, will deteriorate the active control effect.

We established a method using the convolutional neural network to identify the accurate characteristics after the change and compared the control effect before and after. Using our method, we successfully achieved the goal of maintaining the control effect. 

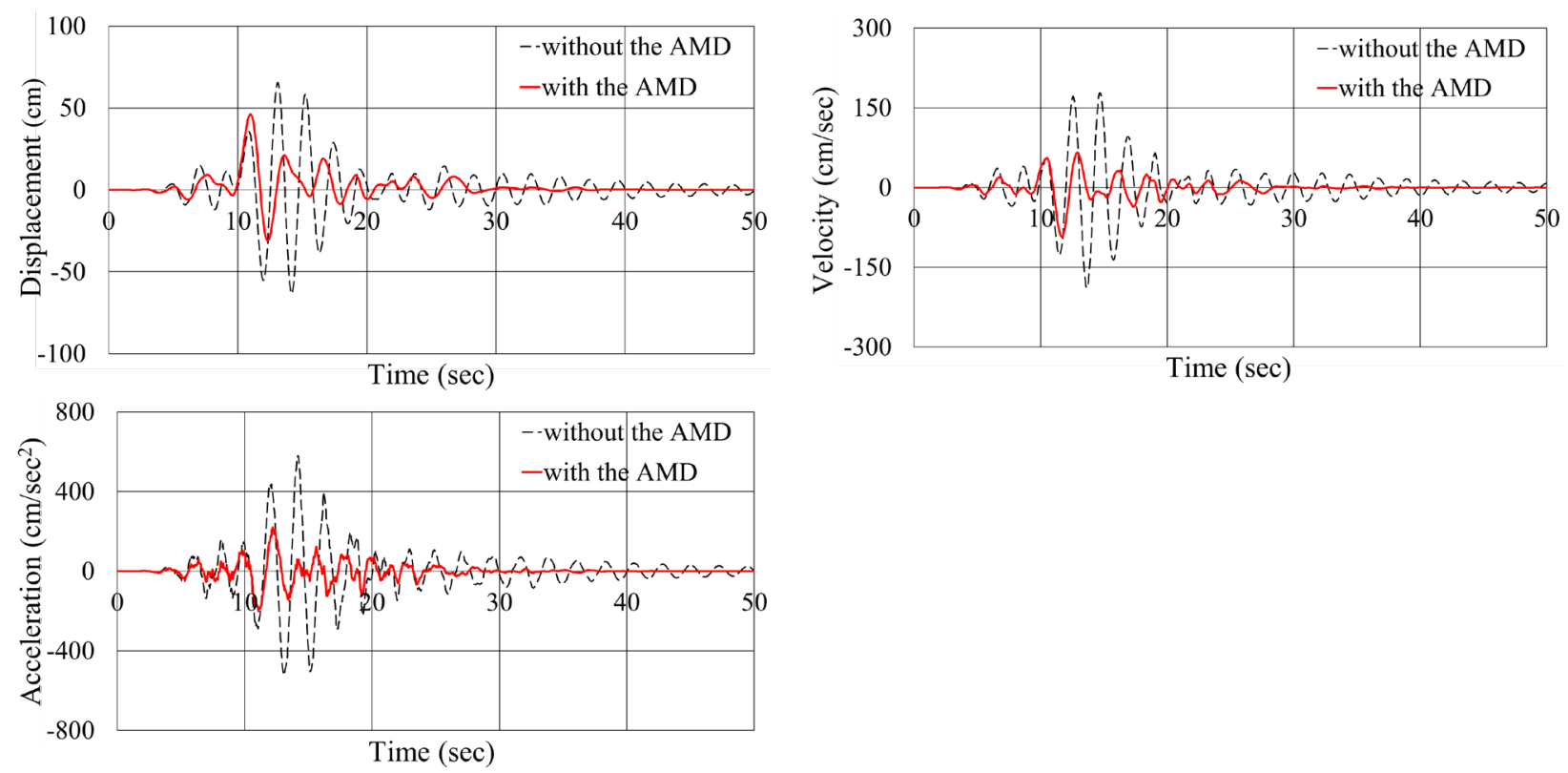

Figure 9 Dynamic response after training

\section{References}

[1] Architectural Institute of Japan, Intelligible Guide to Structural Control, first ed., Maruzen, Tokyo, 2014

[2] Tomohiro MAEDA, Shinta YOSHITOMI and Izuru TAKEWAKI, Stiffness-damping identification of buildings using limited earthquake records and ARX model, J. Struct. Constr. Eng., AIJ, Vol. 76, No.666, 2011, pp. 1415-1423. https://doi.org/10.3130/aijs.76.1415

[3] Hayabe Yasuhiro, Inoue Tetsushiro, Maseki Ryota, Nagashima Ichiro, Ochiai Hiroshi and Saito Kenji, The wind-induced response control by oil dampers and active mass damper: (Part 1) Response control systems and evaluation of wind-induced response performance, Summaries of technical papers of annual meeting, AIJ, 2004, pp. 71-72

[4] Peter Dorato, Luigi Fortuna and G. Muscato, Robust Control for Unstructured Perturbations: An Introduction, Springer Verlag, 1992. https://doi.org/10.1007/BFb0039551

[5] Diederik P. Kingma and Jimmy Lei Ba, Adam: A method for stochastic optimization, the 3rd International Conference for Learning Representations, San Diego, 2015

[6] F. R. Rofooei, Aghababaii Mobarake, G. Ahmadi, Generation of artificial earthquake records with a nonstationary Kanai-Tajimi model, Engineering Structures, Vol. 23, Issue 7, 2001, pp. 827-837. https://doi.org/10.1016/S0141-0296(00)00093-6

[7] Miao CAO, Masato MOTOSAKA, Tsoggerl TSAMBA and Kazushi YOSHIDA, Simulation analysis of a damaged 9-story SRC building during the 2011 Great East Japan Earthquake, Journal of Japan Association for Earthquake Engineering, Vol.13, No.2,2013, pp. 45-64. https://doi.org/10.5610/jaee.13.2_45

[8] IIYAMA Kahori, KURITA Satoshi, MOTOSAKA Masato, CHIBA Kazuki, HIRAMATSU Hiroki and MITSUJI Kazuya, Modal Identification of a Heavily Damaged 9-story SRC Building by Ambient Vibration Measurements, Vol.13, No.2, 2013, pp. 225-242 